\title{
A New Entry to Pd-H Chemistry: Catalytic Asymmetric Conjugate Reduction of Enones with EtOH, and a Highly Enantioselective Synthesis of Warfarin
}

\author{
Yasunori Tsuchiya, Yoshitaka Hamashima, and Mikiko Sodeoka*
}

RIKEN (The Institute of Physical and Chemical Research), 2-1 Hirosawa, Wako 351-0198, Japan

sodeoka@riken.jp

\section{Supporting Information}

\section{List of Contents}

(A) General

(B) Preparation of $\alpha, \beta$-unsaturated ketones and their spectroscopic data

(C) Typical procedure for catalytic asymmetric conjugate reduction of $\alpha, \beta$-unsaturated ketones and analytical data of the reduced products

(D) References

(E) NMR spectra 


\section{(A) General}

All asymmetric reactions were carried out without precaution to exclude air and moisture. Catalysts used in this paper were prepared according to the reported procedure. ${ }^{1}$ NMR spectra were recorded on a JEOL JNM-LA400 spectrometer, operating at $400 \mathrm{MHz}$ for ${ }^{1} \mathrm{H}-\mathrm{NMR}, 100.4 \mathrm{MHz}$ for ${ }^{13} \mathrm{C}-\mathrm{NMR}$. Chemical shifts were reported downfield from TMS $(=0)$ for ${ }^{1} \mathrm{H}-\mathrm{NMR}$. For ${ }^{13} \mathrm{C}-\mathrm{NMR}$, chemical shifts were reported in the scale relative to the solvent used as an internal reference. ${ }^{19} \mathrm{~F}-\mathrm{NMR}$ was measured at $376 \mathrm{MHz}$, and $\mathrm{CF}_{3} \mathrm{COOH}$ was used as external standard. FAB-LRMS was taken on JEOL the Mstation JMS-700 using $m$-nitrobenzyl alcohol (mNBA) as matrix. Optical rotations were measured on a JASCO DIP-370 polarimeter. Column chromatography was performed with silica gel 60 (40-100 $\mu \mathrm{m})$ purchased from KANTO CHEMICAL Co. The enantiomeric excesses (ees) were determined by HPLC analysis. HPLC analysis was performed on JASCO Borwin Ver.1.5 systems consisting of the followings; pump, JASCO PU-2080 plus; detector, JASCO CD-2095 plus measured at $254 \mathrm{~nm}$ or $280 \mathrm{~nm}$; column, DAICEL CHIRALPAK AS-H, IA, and CHIRALCEL OJ-H; mobile phase, hexane/2-propanol (IPA). Dry tetrahydrofuran (THF) was purchased from KANTO CHEMICAL Co. Other reagents were purified by usual methods. 


\section{Representative example}

\section{Preparation of (E)-5-methyl-4-phenyl-3-hexen-2-one (4c)}

\section{Synthesis of ethyl 4-methyl-3-phenylpent-2-enoate ${ }^{2}$}
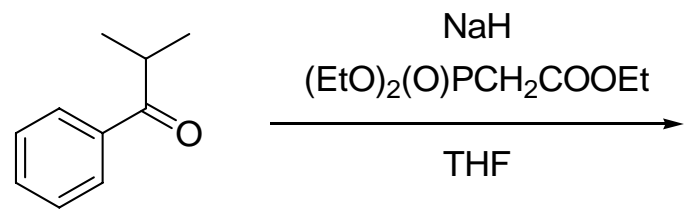

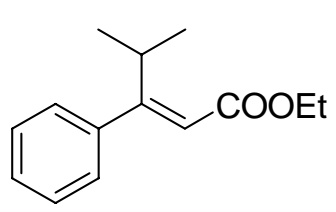

(E)-ester

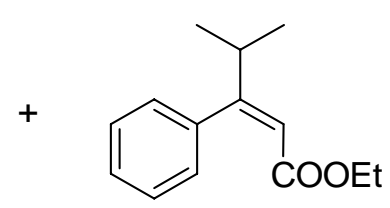

(Z)-ester

To a suspension of $\mathrm{NaH}(20 \mathrm{mmol})$ in THF $(10 \mathrm{~mL})$, a solution of triethyl phosphonoacetate $(5.83$ $\mathrm{g}, 26 \mathrm{mmol}$ ) in THF was added dropwise at $0{ }^{\circ} \mathrm{C}$. The mixture was stirred at room temperature for 30 min. Then, isobutyrophenone $(2.96 \mathrm{~g}, 26 \mathrm{mmol})$ was added to the reaction mixture at $0{ }^{\circ} \mathrm{C}$. After the mixture was stirred at room temperature for $24 \mathrm{~h}$, saturated aqueous sodium bicarbonate $(30 \mathrm{~mL})$ was added for quenching. The mixture was extracted with ethyl acetate $(3 \times 30 \mathrm{~mL})$. The combined organic layers were washed with brine $(30 \mathrm{~mL})$ and dried over anhydrous $\mathrm{Na}_{2} \mathrm{SO}_{4}$. After removal of the solvent, purification by silica gel column chromatography gave $1.85 \mathrm{~g}(8.5 \mathrm{mmol}, 42.5 \%$ yield $)$ of $(E)$-ester as a colorless oil and $1.63 \mathrm{~g}$ (7.4 mmol, 37.0\% yield) of (Z)-ester as a colorless oil (not optimized).

(E)-Ester: ${ }^{1} \mathrm{H}$ NMR $\left(400 \mathrm{MHz}, \mathrm{CDCl}_{3}\right) \delta=7.33-7.10(\mathrm{~m}, 3 \mathrm{H}), 7.22-7.17(\mathrm{~m}, 2 \mathrm{H}), 5.70(\mathrm{~s}, 1 \mathrm{H}), 4.20(\mathrm{q}$, $J=7.1 \mathrm{~Hz}, 2 \mathrm{H}), 4.09(\mathrm{sep}, J=7.1 \mathrm{~Hz}, 1 \mathrm{H}), 1.30$ (t, $J=7.1 \mathrm{~Hz}, 3 \mathrm{H}), 1.09$ (d, $J=7.1 \mathrm{~Hz}, 6 \mathrm{H})$.

(Z)-Ester: ${ }^{1} \mathrm{H}$ NMR (400 MHz, $\left.\mathrm{CDCl}_{3}\right) \delta=7.34-7.24(\mathrm{~m}, 3 \mathrm{H}), 7.11-7.07(\mathrm{~m}, 2 \mathrm{H}), 5.85$ (br s, $\left.1 \mathrm{H}\right), 3.95$ (q, $J=7.1 \mathrm{~Hz}, 2 \mathrm{H}), 2.66(\mathrm{sep}, J=6.8 \mathrm{~Hz}, 1 \mathrm{H}), 1.08(\mathrm{~d}, J=6.8 \mathrm{~Hz}, 6 \mathrm{H}), 1.04(\mathrm{t}, J=7.1 \mathrm{~Hz}, 3 \mathrm{H})$. 
Synthesis of (E)- $N$-methoxy- $N$,4-dimethyl-3-phenylpent-2-enamide ${ }^{3,4}$

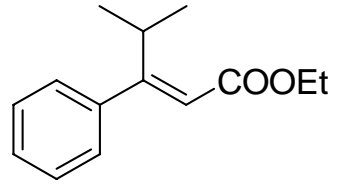

(E)-ester

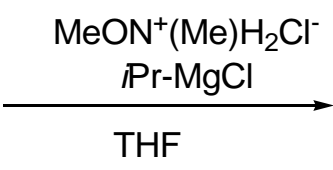

THF

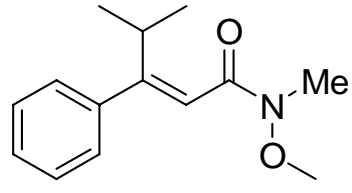

Weinreb amide

To a solution of $(E)$-ester $(1.00 \mathrm{~g}, 4.58 \mathrm{mmol})$ and $\mathrm{N}, \mathrm{O}$-dimethylhydroxyamine hydrochloride $(0.84 \mathrm{~g}, 9.16 \mathrm{mmol})$ in THF $(10 \mathrm{~mL})$, a solution of $2 M \mathrm{THF}$ solution of $i \mathrm{PrMgCl}(10.3 \mathrm{~mL}, 20.6 \mathrm{mmol})$ was added dropwise at $-5{ }^{\circ} \mathrm{C}$. The mixture was stirred for $30 \mathrm{~min}$, and then the mixture was treated with saturated aqueous $\mathrm{NH}_{4} \mathrm{Cl}(30 \mathrm{~mL})$. The mixture was extracted with ethyl acetate, and the organic layer was washed with brine $(30 \mathrm{~mL})$, and dried over anhydrous $\mathrm{Na}_{2} \mathrm{SO}_{4}$. After removal of the solvent, purification by silica gel column chromatography gave $1.07 \mathrm{~g}(4.58 \mathrm{mmol}, 100 \%$ yield $)$ of the corresponding Weinreb amide as a colorless oil: ${ }^{1} \mathrm{H}$ NMR $\left(400 \mathrm{MHz}, \mathrm{CDCl}_{3}\right) \delta=7.36-7.30(\mathrm{~m}, 3 \mathrm{H})$, 7.27-7.20 (m, 2H), 6.09 (br s, 1H), 3.90-4.00 (m, 1H), $3.68(\mathrm{~s}, 3 \mathrm{H}), 3.25(\mathrm{~s}, 3 \mathrm{H}), 1.40(\mathrm{~d}, J=7.1 \mathrm{~Hz}$, $6 \mathrm{H})$.

\section{Synthesis of (E)-5-methyl-4-phenyl-3-hexen-2-one (4c) ${ }^{4}$}<smiles>CON(C)C(=O)/C=C(/c1ccccc1)C(C)C</smiles>

Weinreb amide

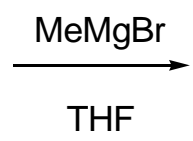

THF

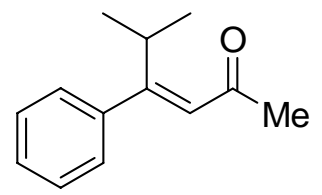

4c

A $1 M$ THF-solution of $\mathrm{MeMgBr}(10 \mathrm{~mL})$ was added dropwise to a solution of the obtained Weinreb amide $(1.07 \mathrm{~g}, 4.58 \mathrm{mmol})$ at $-30{ }^{\circ} \mathrm{C}$. The reaction mixture was stirred at $-5^{\circ} \mathrm{C}$ for $30 \mathrm{~min}$, and then the mixture was treated with saturated aqueous $\mathrm{NH}_{4} \mathrm{Cl}(30 \mathrm{~mL})$. The mixture was extracted with ethyl acetate, and the organic layer was washed with brine $(30 \mathrm{~mL})$, and dried over anhydrous $\mathrm{Na}_{2} \mathrm{SO}_{4}$. After removal of the solvent, purification by silica gel column chromatography afforded $0.847 \mathrm{~g}$ (4.5 mmol, 98.3\% yield) of 5-methyl-4-phenyl-3-hexen-2-one 4c as a colorless oil: ${ }^{1} \mathrm{H}$ NMR (400 MHz, $\left.\mathrm{CDCl}_{3}\right) \delta=7.35-7.31(\mathrm{~m}, 3 \mathrm{H}), 7.22-7.19(\mathrm{~m}, 2 \mathrm{H}), 6.07(\mathrm{~s}, 1 \mathrm{H}), 4.03(\mathrm{sep}, J=7.1 \mathrm{~Hz}, 1 \mathrm{H}), 2.25(\mathrm{~s}, 3 \mathrm{H})$, 
$1.07(\mathrm{~d}, J=7.1 \mathrm{~Hz}, 6 \mathrm{H}) ;{ }^{13} \mathrm{C} \mathrm{NMR}\left(100 \mathrm{MHz}, \mathrm{CDCl}_{3}\right) \delta=198.9,165.5,140.9,127.8,127.6,127.6$, $125.9,32.3,29.5,21.3$

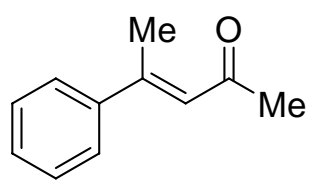

(E)-4-Phenyl-3-penten-2-one (4a): colorless oil; ${ }^{1} \mathrm{H}$ NMR $\left(400 \mathrm{MHz}, \mathrm{CDCl}_{3}\right) \delta=$ 7.51-7.46 (m, 2H), 7.40-7.36 (m, 3H), 6.52 (s, 1H), 2.54 (br s, 3H), 2.30 (d, $J=0.7$ $\mathrm{Hz}, 3 \mathrm{H}) ;{ }^{13} \mathrm{C} \mathrm{NMR}\left(100 \mathrm{MHz}, \mathrm{CDCl}_{3}\right) \delta=198.9,153.9,142.5,129.1,128.5,126.5,124.5,32.2,18.3$.<smiles>CC/C(=C\C(C)=O)c1ccccc1</smiles>

(E)-4-Phenyl-3-hexen-2-one (4b): colorless oil; ${ }^{1} \mathrm{H}$ NMR $\left(400 \mathrm{MHz}, \mathrm{CDCl}_{3}\right) \delta=$ 7.50-7.42 (m, 2H), 7.40-7.34 (m, 3H), $6.39(\mathrm{~s}, 1 \mathrm{H}), 3.05$ (q, J=7.6 Hz, 2H), 2.28 (s, $3 \mathrm{H}), 1.06(\mathrm{t}, J=7.6 \mathrm{~Hz}, 3 \mathrm{H}) ;{ }^{13} \mathrm{C}$ NMR $\left(100 \mathrm{MHz}, \mathrm{CDCl}_{3}\right) \delta=198.4,160.4,141.4,128.9,128.5,126.8$, $124.1,32.3,24.4,13.5$.

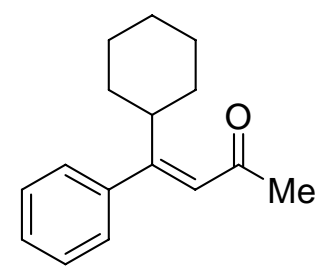

(E)-4-Cyclohexyl-4-phenyl-3-buten-2-one (4d): colorless oil; ${ }^{1} \mathrm{H}$ NMR (400 MHz, $\left.\mathrm{CDCl}_{3}\right) \delta=7.36-7.29(\mathrm{~m}, 3 \mathrm{H}), 7.21-7.14(\mathrm{~m}, 2 \mathrm{H}), 6.03(\mathrm{~s}, 1 \mathrm{H}), 3.66(\mathrm{tt}, J=12.2$, $2.9 \mathrm{~Hz}, 1 \mathrm{H}), 2.24(\mathrm{~s}, 3 \mathrm{H}), 1.71-1.57(\mathrm{~m}, 5 \mathrm{H}), 1.44-1.32(\mathrm{~m}, 2 \mathrm{H}), 1.30-1.26(\mathrm{~m}, 2 \mathrm{H})$, 1.14-0.98 (m, 1H); ${ }^{13} \mathrm{C}$ NMR (100 MHz, $\left.\mathrm{CDCl}_{3}\right) \delta=198.8,165.1,141.5,127.7,127.5,127.4,126.0$, $40.5,32.2,31.5,26.3,25.9$.

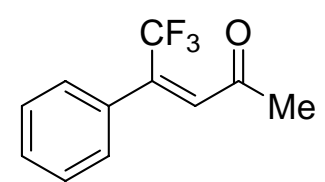

(E)-4-Trifluoromethyl-4-phenyl-3-buten-2-one (4e): colorless oil; ${ }^{1} \mathrm{H}$ NMR $\left(\mathrm{CDCl}_{3}, \mathrm{TMS}\right) \delta=7.44-7.40(\mathrm{~m}, 5 \mathrm{H}), 6.45(\mathrm{~s}, 1 \mathrm{H}), 2.42(\mathrm{~s}, 3 \mathrm{H}) ;{ }^{13} \mathrm{C} \mathrm{NMR}(100$ $\left.\mathrm{MHz}, \mathrm{CDCl}_{3}\right) \delta=199.7,136.2\left(\mathrm{q}, J_{C-F}=3.3 \mathrm{~Hz}\right), 134.6\left(\mathrm{q}, J_{C-F}=31.2 \mathrm{~Hz}\right) 133.3,129.4,128.6,127.7$, $122.5\left(\mathrm{q}, J_{C-F}=274.8 \mathrm{~Hz}\right), 30.5 ;{ }^{19} \mathrm{~F} \operatorname{NMR}\left(376 \mathrm{MHz}, \mathrm{CDCl}_{3}\right) \delta=17.0$.

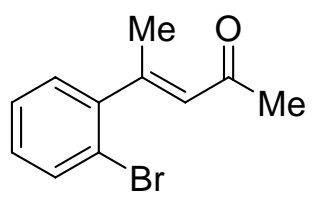

(E)-4-(2-Bromophenyl)-3-buten-2-one (4f): colorless oil; ${ }^{1} \mathrm{H}$ NMR (400 MHz, $\left.\mathrm{CDCl}_{3}\right) \delta=7.58(\mathrm{dd}, J=7.8,1.0 \mathrm{~Hz}, 1 \mathrm{H}), 7.30(\mathrm{dt}, J=7.8,1.0 \mathrm{~Hz}, 1 \mathrm{H}), 7.22-7.12$ $(\mathrm{m}, 2 \mathrm{H}), 6.18(\mathrm{br} \mathrm{s}, 1 \mathrm{H}), 2.44(\mathrm{~d}, J=1.2 \mathrm{~Hz}, 3 \mathrm{H}), 2.28(\mathrm{~s}, 3 \mathrm{H}) ;{ }^{13} \mathrm{C} \mathrm{NMR}\left(100 \mathrm{MHz}, \mathrm{CDCl}_{3}\right) \delta=172.4$, $155.0,144.8,133.1,129.1,128.8,127.4,127.4,120.7,32.1,20.7$. 
(C) Typical procedure for catalytic asymmetric conjugate reduction of $\alpha, \beta$-unsaturated ketones and analytical data of the reduced products

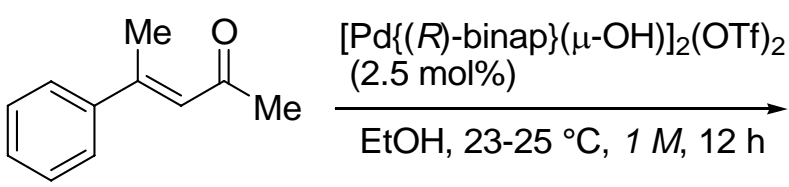

$4 a$

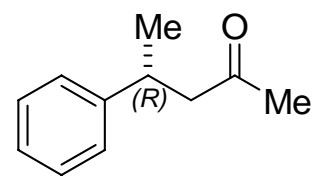

$5 a$

Typical procedure: The solution of $[\operatorname{Pd}\{(R)-\operatorname{binap}\}(\mu-\mathrm{OH})]_{2}(\mathrm{OTf})_{2}(4.5 \mathrm{mg}, 0.0025 \mathrm{mmol})$ and $(E)-4-$ phenyl-3-penten-2-one (4a) $(16.0 \mathrm{mg}, 0.1 \mathrm{mmol})$ in ethanol $(0.1 \mathrm{~mL})$ was stirred at ambient temperature for $12 \mathrm{~h}$. Evaporation of the solvent, followed by silica gel chromatography gave 4-phenyl-pentan-2-one $((R)-5 a)$ in $>99 \%$ yield $(16.1 \mathrm{mg}, 0.099 \mathrm{mmol})$ with $74 \%$ ee as a colorless oil. The ee was determined by chiral HPLC analysis: DAICEL CHIRALPAK AS-H, flow rate: $0.5 \mathrm{~mL} / \mathrm{min}, \mathrm{UV}=280 \mathrm{~nm}$, Hex:IPA $=98: 2, \tau_{\text {minor }}=12.0 \mathrm{~min}, \tau_{\text {major }}=13.6 \mathrm{~min} ;{ }^{1} \mathrm{H} \mathrm{NMR}\left(400 \mathrm{MHz}, \mathrm{CDCl}_{3}\right) \delta=7.32-7.25(\mathrm{~m}, 2 \mathrm{H})$, 7.23-7.14 (m, 3H), 3.27 (ddq, $J=6.6,7.8,6.8 \mathrm{~Hz}, 1 \mathrm{H}), 2.76(\mathrm{dd}, J=16.4,6.6 \mathrm{~Hz}, 1 \mathrm{H}), 2.66(\mathrm{dd}, J=$ 16.4, 7.8 Hz, 1H), 2.07 (s, 3H), 1.27 (d, $J=6.8 \mathrm{~Hz}, 3 \mathrm{H}) ;{ }^{13} \mathrm{C} \mathrm{NMR}\left(100 \mathrm{MHz}, \mathrm{CDCl}_{3}\right) \delta=207.9,146.2$, $128.5,126.8,126.3,52.0,35.4,30.8,22.0 ;[\alpha]_{D}^{26}=-38.0\left(\mathrm{c}=0.5, \mathrm{CHCl}_{3}\right)(74 \%$ ee $) .\left\{\right.$ Lit. $^{4}[\alpha]_{D}^{25}=-49.5$ $\left(\mathrm{c}=1.0, \mathrm{CHCl}_{3}\right)(95 \%$ ee, $R$ enantiomer $\left.)\right\}$.

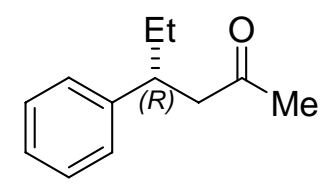

4-Phenyl-hexan-2-one ((R)-5b): colorless oil; 97\% yield, 84\% ee. The ee was determined by chiral HPLC analysis: DAICEL CHIRALCEL OJ-H, flow rate: 0.5 $\mathrm{mL} / \mathrm{min}, \mathrm{UV}=280 \mathrm{~nm}, \mathrm{Hex}: \mathrm{IPA}=99: 1, \tau_{\text {minor }}=20.7 \mathrm{~min}, \tau_{\text {major }}=24.4 \mathrm{~min} ;{ }^{1} \mathrm{H} \mathrm{NMR}(400 \mathrm{MHz}$, $\left.\mathrm{CDCl}_{3}\right) \delta=7.32-7.25(\mathrm{~m}, 2 \mathrm{H}), 7.24-7.14(\mathrm{~m}, 3 \mathrm{H}), 3.03(\mathrm{dddd}, J=7.3,7.1,7.6,7.4 \mathrm{~Hz}, 1 \mathrm{H}), 2.74(\mathrm{dd}, J$ $=16.6,7.3 \mathrm{~Hz}, 1 \mathrm{H}), 2.70(\mathrm{dd}, J=16.6,7.1 \mathrm{~Hz}, 1 \mathrm{H}), 2.01(\mathrm{~s}, 3 \mathrm{H}), 1.67(\mathrm{ddq}, J=12.9,7.6,7.3 \mathrm{~Hz}, 1 \mathrm{H})$, $1.55(\mathrm{ddq}, J=12.9,7.4,7.3 \mathrm{~Hz}, 1 \mathrm{H}), 0.77(\mathrm{t}, J=7.3 \mathrm{~Hz}, 3 \mathrm{H}) ;{ }^{13} \mathrm{C} \mathrm{NMR}\left(100 \mathrm{MHz}, \mathrm{CDCl}_{3}\right) \delta=208.0$, $144.2,128.4,127.5,126.3,50.5,42.9,30.6,29.3,11.9 ;[\alpha]_{D}^{26}=-30.0(\mathrm{c}=1.7, \mathrm{EtOH})(84 \%$ ee $) .\left\{\right.$ Lit. $^{5}:$ $[\alpha]_{D}^{20}=-28.7(\mathrm{c}=1.0, \mathrm{EtOH})(87 \%$ ee, $R$ enantiomer $\left.)\right]$ 
5-Methyl-4-phenyl-hexan-2-one ((S)-5c): colorless oil; >99\% yield, 92\% ee. The ee was determined by chiral HPLC analysis: DAICEL CHIRALCEL OJ-H, flow rate: $0.5 \mathrm{~mL} / \mathrm{min}, \mathrm{UV}=280 \mathrm{~nm}$, Hex:IPA $=99: 1, \tau_{\text {minor }}=20.6 \mathrm{~min}, \tau_{\text {major }}=25.4$ $\min ;{ }^{1} \mathrm{H}$ NMR $\left(400 \mathrm{MHz}, \mathrm{CDCl}_{3}\right) \delta=7.26-7.15(\mathrm{~m}, 2 \mathrm{H}), 7.17-7.10(\mathrm{~m}, 3 \mathrm{H}), 2.89$ (ddd, $J=9.0,7.6,5.9$ Hz, 1H), 2.79 (dd, $J=15.9,5.9 \mathrm{~Hz}, 1 \mathrm{H}), 2.75$ (dd, $J=15.9,9.0 \mathrm{~Hz}, 1 \mathrm{H}), 1.95$ (s, 3H), 1.80 (dsep, $J=$ 7.6, $6.6 \mathrm{~Hz}, 1 \mathrm{H}), 0.90(\mathrm{~d}, J=6.6 \mathrm{~Hz}, 3 \mathrm{H}), 0.72(\mathrm{~d}, J=6.6 \mathrm{~Hz}, 3 \mathrm{H}) ;{ }^{13} \mathrm{C} \mathrm{NMR}\left(100 \mathrm{MHz}, \mathrm{CDCl}_{3}\right) \delta=$ $208.3,143.2,128.2,128.1,126.2,48.0,47.6,33.3,30.6,20.7,20.3 ;[\alpha]_{D}^{26}=-37.3\left(\mathrm{c}, 0.5=\mathrm{CHCl}_{3}\right)(92 \%$ ee). $\left\{\right.$ Lit. $^{4}[\alpha]_{D}^{24}=-38.9\left(\mathrm{c}=0.97, \mathrm{CHCl}_{3}\right)(98 \%$ ee, $S$ enantiomer $\left.)\right\}$.

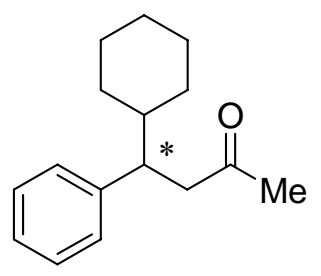

4-Cyclohexyl-4-phenyl-butan-2-one (5d): white solid; 97\% yield, 86\% ee. The ee was determined by chiral HPLC analysis: DAICEL CHIRALCEL OJ-H, flow rate: $0.5 \mathrm{~mL} / \mathrm{min}, \mathrm{UV}=280 \mathrm{~nm}, \mathrm{Hex}: \mathrm{IPA}=98: 2, \tau_{\text {major }}=16.1 \mathrm{~min}, \tau_{\text {minor }}=19.8 \mathrm{~min} ;{ }^{1} \mathrm{H}$ NMR (400 MHz, $\left.\mathrm{CDCl}_{3}\right) \delta=7.29-7.23(\mathrm{~m}, 2 \mathrm{H}), 7.20-7.10(\mathrm{~m}, 1 \mathrm{H})$, 7.15-7.10 (m, 2H), 2.97-2.90 (m, 1H), $2.84(\mathrm{dd}, J=15.9,5.4 \mathrm{~Hz}, 1 \mathrm{H}), 2.74(\mathrm{dd}, J=15.9,9.2 \mathrm{~Hz}, 1 \mathrm{H}), 1.97(\mathrm{~s}, 3 \mathrm{H}), 1.79-1.70(\mathrm{~m}, 2 \mathrm{H})$, 1.65-1.59 (m, 2H), 1.50-1.40 (m, 2H), 1.26-1.10 (m, 1H), 1.12-1.02 (m, 2H), 1.02-0.89 (m, 1H), 0.85$0.70(\mathrm{~m}, 1 \mathrm{H}) ;{ }^{13} \mathrm{C} \mathrm{NMR}\left(100 \mathrm{MHz}, \mathrm{CDCl}_{3}\right) \delta=208.5,143.4,128.3,128.1,126.2,47.5,47.2,47.1,43.0$, $31.1,30.7,30.6,26.5,26.3 ;[\alpha]_{D}^{26}=-26.7\left(\mathrm{c}=0.7, \mathrm{CHCl}_{3}\right)(86 \%$ ee $) .\left\{\right.$ Lit. $^{6}[\alpha]_{D}^{23}=-23.0(\mathrm{c}=0.13$, $\left.\mathrm{CHCl}_{3}\right)(77 \%$ ee $\left.)\right\}$

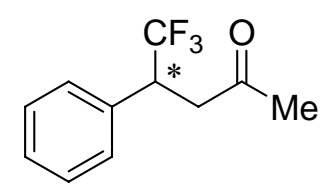

4-Phenyl-4-trifluoromethyl-butan-2-one (5e): colorless oil; 85\% yield, 84\% ee. The ee was determined by chiral HPLC analysis: DAICEL CHIRALCEL OJ-H, flow rate: $0.5 \mathrm{~mL} / \mathrm{min}, \mathrm{UV}=280 \mathrm{~nm}, \mathrm{Hex}: \mathrm{IPA}=7: 1, \tau_{\text {minor }}=20.3 \mathrm{~min}, \tau_{\text {major }}=24.3$ $\min ;{ }^{1} \mathrm{H}$ NMR $\left(400 \mathrm{MHz}, \mathrm{CDCl}_{3}\right) \delta=7.35-7.20(\mathrm{~m}, 5 \mathrm{H}), 4.01\left(\mathrm{ddq}, J=8.1,5.4 \mathrm{~Hz}, J_{H-F}=7.5 \mathrm{~Hz}, 1 \mathrm{H}\right)$, $3.11(\mathrm{dd}, J=17.8,5.4 \mathrm{~Hz}, 1 \mathrm{H}), 3.01(\mathrm{dd}, J=17.8,8.1 \mathrm{~Hz}, 1 \mathrm{H}), 2.11(\mathrm{~s}, 3 \mathrm{H}) ;{ }^{13} \mathrm{C} \mathrm{NMR}(100 \mathrm{MHz}$, $\left.\mathrm{CDCl}_{3}\right) \delta=203.6,134.4,128.9,128.7,128.3,126.7\left(\mathrm{q}, J_{C-F}=278.1 \mathrm{~Hz}\right), 44.5\left(\mathrm{q}, J_{C-F}=27.2 \mathrm{~Hz}\right), 43.0$ 
$\left(\mathrm{d}, J_{C-F}=16 \mathrm{~Hz}\right), 30.3 ;{ }^{19} \mathrm{~F}$ NMR $\left(376 \mathrm{MHz}, \mathrm{CDCl}_{3}\right) \delta=6.07\left(\mathrm{~d}, J_{H-F}=7.5 \mathrm{~Hz}\right) ;$ FAB-LRMS $(m N B A)$ $\mathrm{m} / \mathrm{z} 217[\mathrm{M}+1]^{+}, 216[\mathrm{M}]^{+} ;[\alpha]_{D}^{26}=-43.1\left(\mathrm{c}=0.45, \mathrm{CHCl}_{3}\right)(84 \%$ ee $)$.

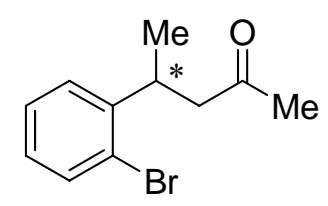

4-(2-Bromophenyl)-pentan-2-one (5f): colorless oil; $>99 \%$ yield, $80 \%$ ee. The ee was determined by chiral HPLC analysis: DAICEL CHIRALCEL OJ-H, flow rate: $0.5 \mathrm{~mL} / \mathrm{min}, \mathrm{UV}=280 \mathrm{~nm}, \mathrm{Hex}: \mathrm{IPA}=7: 1, \tau_{\text {minor }}=24.6 \mathrm{~min}, \tau_{\text {major }}=26.4 \mathrm{~min} ;{ }^{1} \mathrm{H}$ $\operatorname{NMR}\left(400 \mathrm{MHz}, \mathrm{CDCl}_{3}\right) \delta=7.55(\mathrm{~d}, J=8.0 \mathrm{~Hz}, 1 \mathrm{H}), 7.30-7.20(\mathrm{~m}, 2 \mathrm{H}), 7.06(\mathrm{dt}, J=2.0,7.6,1 \mathrm{H})$, $3.78(\mathrm{ddq}, J=8.8,5.4,6.8 \mathrm{~Hz}, 1 \mathrm{H}), 2.80(\mathrm{dd}, J=16.4$, and $5.4 \mathrm{~Hz}, 1 \mathrm{H}), 2.59(\mathrm{dd}, J=16.4$, and $8.8 \mathrm{~Hz}$, $1 \mathrm{H}), 2.13(\mathrm{~s}, 3 \mathrm{H}), 1.26(\mathrm{~d}, J=6.8 \mathrm{~Hz}, 3 \mathrm{H}) ;{ }^{13} \mathrm{C} \mathrm{NMR}\left(100 \mathrm{MHz}, \mathrm{CDCl}_{3}\right) \delta=207.2,144.6,133.1,127.8$, $127.7,127.1,124.2,50.8,34.1,30.0,20.4 ;$ FAB-LRMS $(m N B A) \mathrm{m} / \mathrm{z} 242[\mathrm{M}+2]^{+}, 240[\mathrm{M}]^{+} ;[\alpha]_{D}^{26}=-$ $32.0\left(\mathrm{c}=0.15, \mathrm{CHCl}_{3}\right)(80 \% \mathrm{ee})$.

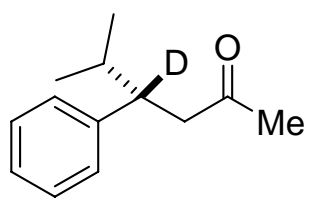

5-Methyl-4-deuterium-4-phenyl-hexan-2-one (7): colorless oil; $>99 \%$ yield, 86\% ee. The reaction was carried out according to the general procedure using $\mathrm{CH}_{3} \mathrm{CD}_{2} \mathrm{OH}$ (1,1-d-ethanol) as a solvent. The ee was determined by chiral HPLC analysis: DAICEL CHIRALCEL OJ-H, flow rate: $0.5 \mathrm{~mL} / \mathrm{min}, \mathrm{UV}=280 \mathrm{~nm}, \mathrm{Hex}: \mathrm{IPA}=99: 1, \tau_{\text {minor }}=$ $20.6 \mathrm{~min}, \tau_{\text {major }}=25.4 \mathrm{~min} ;{ }^{1} \mathrm{H} \operatorname{NMR}\left(400 \mathrm{MHz}, \mathrm{CDCl}_{3}\right) \delta=7.29-7.24(\mathrm{~m}, 2 \mathrm{H}), 7.20-7.12(\mathrm{~m}, 3 \mathrm{H})$, 2.85-2.73 (m, 2H), $1.98(\mathrm{~s}, 3 \mathrm{H}), 1.82(\mathrm{sep}, J=6.6 \mathrm{~Hz}, 1 \mathrm{H}), 0.93(\mathrm{~d}, J=6.6 \mathrm{~Hz}, 3 \mathrm{H}), 0.74(\mathrm{~d}, J=6.6 \mathrm{~Hz}$, $3 \mathrm{H})$. The absolute stereochemistry was tentatively assigned by analogy to that of $\mathbf{5 c}$.

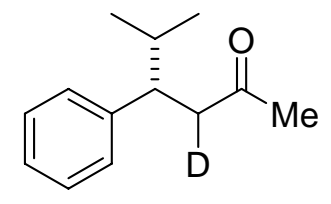

5-Methyl-3-deuterium-4-phenyl-hexan-2-one (8): colorless oil, 81\% yield, $84 \%$ ee. The reaction was carried out according to the general procedure using $\mathrm{CH}_{3} \mathrm{CH}_{2} \mathrm{OD}$ (d-ethanol) as a solvent. The ee was determined by chiral HPLC analysis: DAICEL CHIRALCEL OJ-H, flow rate: $0.5 \mathrm{~mL} / \mathrm{min}, \mathrm{UV}=280 \mathrm{~nm}, \mathrm{Hex}: \mathrm{IPA}=99: 1, \tau_{\text {minor }}=$ $20.6 \mathrm{~min}, \tau_{\text {major }}=25.4 \mathrm{~min} ;{ }^{1} \mathrm{H}$ NMR $\left(400 \mathrm{MHz}, \mathrm{CDCl}_{3}\right) \delta=7.29-7.24(\mathrm{~m}, 2 \mathrm{H}), 7.20-7.10(\mathrm{~m}, 3 \mathrm{H})$, 2.95-2.84 (m, 1H), 2.84-2.70 (m, 1H), 1.97 (s, 3H), $1.83(\mathrm{dsep}, J=6.6,6.8 \mathrm{~Hz}, 1 \mathrm{H}), 0.93$ (d, $J=6.6 \mathrm{~Hz}$, 
$3 \mathrm{H}), 0.74(\mathrm{~d}, J=6.8 \mathrm{~Hz}, 3 \mathrm{H})$. The absolute stereochemistry was tentatively assigned by analogy to that of $5 c$.

Competitive experiment between enone 4a and ester 6: The mixture of enone $4 \mathbf{a}(0.1 \mathrm{mmol})$ and the ester $6(0.1 \mathrm{mmol})$ in ethanol $(0.1 \mathrm{~mL})$ in the presence of the Pd-complex $3(2.5 \mathrm{~mol} \%)$ was stirred at ambient temperature. After $12 \mathrm{~h}$, the solvent was removed. The NMR-spectra of the crude mixture were shown in Section E (S-19).

\section{Synthesis of warfarin ${ }^{7}$}

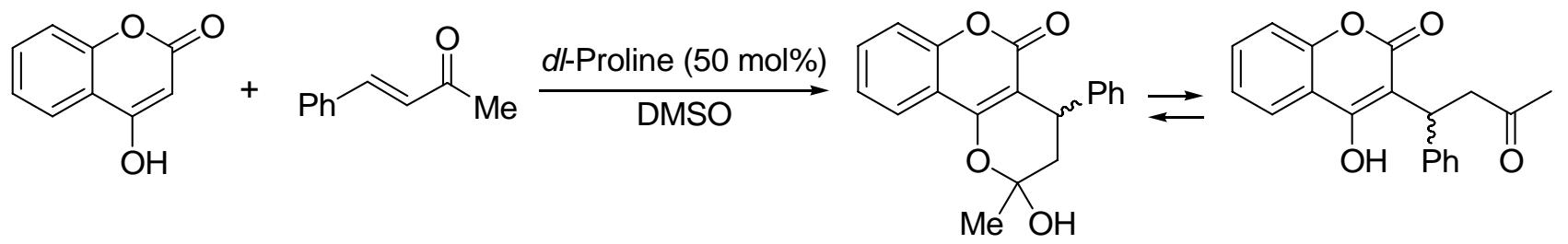

To a stirred solution of benzalacetone $(1.62 \mathrm{~g}, 10 \mathrm{mmol})$ and 4-hydroxycoumarin $(1.46 \mathrm{~g}, 10$ mmol) in DMSO $(20 \mathrm{~mL})$ was added $d \mathrm{l}$-proline $(0.58 \mathrm{~g}, 5 \mathrm{mmol})$ and the mixture was stirred at ambient temperature. After $24 \mathrm{~h}$, the mixture was extracted with $\mathrm{CH}_{2} \mathrm{Cl}_{2}$, and the combined organic extract was washed with $\mathrm{H}_{2} \mathrm{O}$. Evaporation of the solvent and purification by silica gel chromatography gave racwarfarin $(2.45 \mathrm{~g}, 0.80 \mathrm{mmol})$ in $80 \%$ yield as a white solid.

\section{Synthesis of Dehydrowarfarin ${ }^{8}$}<smiles>C=CC(C)=CC(=Cc1c(O)c2ccccc2oc1=O)c1ccccc1-c1ccccc1</smiles>

Copper (I) chloride $(2.56 \mathrm{~g}, 24.5 \mathrm{mmol})$ was added to a stirred solution of rac-warfarin $(5.05 \mathrm{~g}$, $16.3 \mathrm{mmol})$ in anhydrous pyridine $(30 \mathrm{~mL})$. The mixture was stirred at $55-60{ }^{\circ} \mathrm{C}$, while being aerated with steady flow of air. After $6 \mathrm{~h}$, the reaction mixture was cooled to ambient temperature and diluted with $\mathrm{H}_{2} \mathrm{O}(150 \mathrm{~mL})$ and acidified $(2 \mathrm{M} \mathrm{HCl}, \mathrm{pH}=1)$. The mixture was extracted with AcOEt, and the 
combined organic extract was washed with $\mathrm{H}_{2} \mathrm{O}$. Removal of the solvent gave the crude product quantitatively, which was used without further purification.

\section{Synthesis of 4-methyl-warfarin (11) ${ }^{9}$}

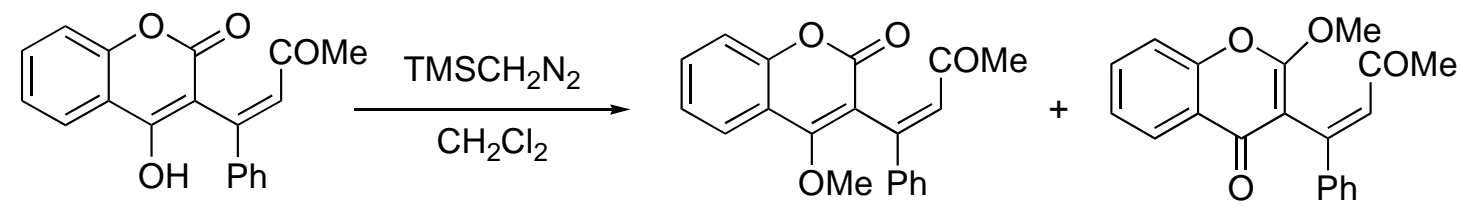

To a solution of dehydrowarfarin $(200 \mathrm{mg}, 0.65 \mathrm{mmol})$ in $\mathrm{CH}_{2} \mathrm{Cl}_{2}(5 \mathrm{~mL})$ was added dropwise excess of $2 M$-trimethylsilyldiazomethane in diethyether at $0{ }^{\circ} \mathrm{C}$. After stirring for $1 \mathrm{~h}$, solvent was removed, and purification by silica gel chromatography gave 4-methyl-warfarin as a major product (179.4 $\mathrm{mg}, 0.56 \mathrm{mmol})$ and 2-methyl-warfarin $(25.6 \mathrm{mg}, 0.08 \mathrm{mmol})$ as a minor product. These compounds were found identical by comparison of ${ }^{1} \mathrm{H}$ NMR with the reported values.<smiles>COc1c(/C(=C\C(C)C)c2ccccc2)c(=O)oc2ccccc12</smiles>

4-Methyl-dehydrowarfarin (11): ${ }^{1} \mathrm{H}$ NMR $\left(400 \mathrm{MHz}, \mathrm{CDCl}_{3}\right) \delta=7.75(\mathrm{~d}, J=$ $7.6 \mathrm{~Hz}, 1 \mathrm{H}), 7.61(\mathrm{t}, J=8.7 \mathrm{~Hz}, 1 \mathrm{H}), 7.55-7.30(\mathrm{~m}, 7 \mathrm{H}), 7.01(\mathrm{~s}, 1 \mathrm{H}), 3.88(\mathrm{~s}$, $3 \mathrm{H}), 2.32(\mathrm{~s}, 3 \mathrm{H})$.<smiles>COc1oc2ccccc2c(=O)c1/C(=C\C(C)C)c1ccccc1</smiles>

2-Methyl-dehydrowarfarin: ${ }^{1} \mathrm{H}$ NMR $\left(400 \mathrm{MHz}, \mathrm{CDCl}_{3}\right) \delta=8.21(\mathrm{~d}, J=7.6$ $\mathrm{Hz}, 1 \mathrm{H}), 7.45(\mathrm{t}, J=8.7 \mathrm{~Hz}, 1 \mathrm{H}), 7.55-7.30(\mathrm{~m}, 7 \mathrm{H}), 6.72(\mathrm{~s}, 1 \mathrm{H}), 3.98(\mathrm{~s}, 3 \mathrm{H})$, $2.14(\mathrm{~s}, 3 \mathrm{H})$.

\section{Conjugate reduction of 4-methyl-warfarin}<smiles>COc1c(/C(=C\C(C)=O)c2ccccc2)c(=O)oc2ccccc12</smiles>

11

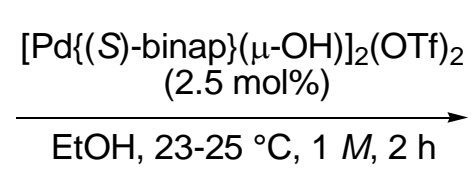

$\mathrm{EtOH}, 23-25^{\circ} \mathrm{C}, 1 \mathrm{M}, 2 \mathrm{~h}$

The solution of $[\operatorname{Pd}\{(S) \text {-binap }\}(\mu-\mathrm{OH})]_{2}(\mathrm{OTf})_{2}(4.5 \mathrm{mg}, 0.0025 \mathrm{mmol})$ and $(E)-4-m e t h y l-d e h y d r o-$ warfarin (11) $(32.0 \mathrm{mg}, 0.1 \mathrm{mmol})$ in ethanol $(0.1 \mathrm{~mL})$ was stirred at ambient temperature for $2 \mathrm{~h}$. After evaporation, purification by silica gel chromatography gave $(S)-4-$ methyl-warfarin (12) in quantitative 
yield with $96 \%$ ee as a colorless oil. The ee was determined by chiral HPLC analysis: DAICEL CHIRALPAK AS-H, flow rate: $1.0 \mathrm{~mL} / \mathrm{min}, \mathrm{UV}=254 \mathrm{~nm}, \mathrm{Hex}: \mathrm{IPA}=7: 1, \tau_{\text {minor }}=11.1 \mathrm{~min}, \tau_{\text {major }}=$ $13.9 \mathrm{~min} ;{ }^{1} \mathrm{H} \mathrm{NMR}\left(400 \mathrm{MHz}, \mathrm{CDCl}_{3}\right) \delta=7.67(\mathrm{dd}, J=8.0,1.4 \mathrm{~Hz}, 1 \mathrm{H}), 7.47($ br d, $J=8.0 \mathrm{~Hz}, 3 \mathrm{H})$, 7.32-7.22 (m, 4H), 7.18 (br t, $J=7.3 \mathrm{~Hz}, 1 \mathrm{H}), 4.98$ (dd, $J=9.5,5.6 \mathrm{~Hz}, 1 \mathrm{H}), 4.05$ (s, 3H), 3.89 (dd, $J=$ $18.1,9.5 \mathrm{~Hz}, 1 \mathrm{H}), 3.22(\mathrm{dd}, J=18.1,5.6 \mathrm{~Hz}, 1 \mathrm{H}), 2.18(\mathrm{~s}, 3 \mathrm{H}) ;{ }^{13} \mathrm{C} \mathrm{NMR}\left(100 \mathrm{MHz}, \mathrm{CDCl}_{3}\right) \delta=207.6$, $164.4,153.1,142.1,131.5,128.5,127.9,126.7,124.0,123.6,119.6,116.8,116.7,62.0,45.8,36.3,30.3$. $[\alpha]_{D}^{24}=+48.2(\mathrm{c}=1.45, \mathrm{EtOH})$.

\section{Demethylation of 4-methyl-warfarin}<smiles>COc1c([C](CCC(C)=O)c2ccccc2)c(=O)oc2ccccc12</smiles>

12

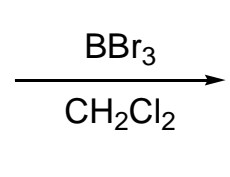

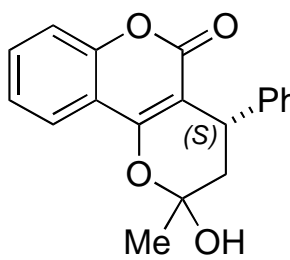

13 (ketal-form)

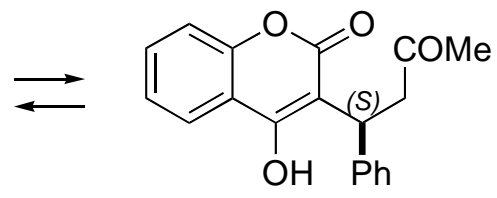

13 (enol-form)

$1 M \mathrm{BBr}_{3}$ in $\mathrm{CH}_{2} \mathrm{Cl}_{2}$ solution was added dropwise to the solution of (S)-4-methyl-warfarin (12) (32.0 $\mathrm{mg}, 0.1 \mathrm{mmol})$ in $\mathrm{CH}_{2} \mathrm{Cl}_{2}(2 \mathrm{~mL})$ at $-78{ }^{\circ} \mathrm{C}$. The resulting mixture was gradually warmed to $0{ }^{\circ} \mathrm{C}$ over 6 h. ${ }^{10}$ After $6 \mathrm{~h}$, the reaction mixture was quenched with saturated aqueous $\mathrm{Na}_{2} \mathrm{CO}_{3}$ and extracted with AcOEt $(20 \mathrm{~mL} \times 3)$. Removal of the dried solvent (anhydrous $\left.\mathrm{Na}_{2} \mathrm{SO}_{4}\right)$, followed by purification by silica gel chromatography gave (S)-warfarin $(24.4 \mathrm{mg}, 0.079 \mathrm{mmol})$ in $79.2 \%$ yield with $96 \%$ ee as a white solid. A single recrystallization in acetone/water furnished optically pure (S)-warfarin in $91 \%$ yield with $>99 \%$ ee. The ee was determined by chiral HPLC analysis: DAICEL CHIRALCEL IA, flow rate: $1.0 \mathrm{~mL} / \mathrm{min}, \mathrm{UV}=254 \mathrm{~nm}$, Hex:IPA $=3: 1(0.1 \% \mathrm{v} / \mathrm{v}$ TFA $), \tau_{\text {minor }}=4.8 \mathrm{~min}, \tau_{\text {major }}=8.8 \mathrm{~min} ;{ }^{1} \mathrm{H}$ NMR (400 MHz, $\left.\mathrm{CDCl}_{3}\right) \delta=9.49(\mathrm{br} \mathrm{s}, 0.1 \mathrm{H}$, enol), $7.94(\mathrm{dd}, J=8.0,1.7 \mathrm{~Hz}, 0.1 \mathrm{H}$, enol), 7.90 (dd, $J=$ 8.1, $1.7 \mathrm{~Hz}, 0.45 \mathrm{H}$, ketal), 7.80 (br d, $J=8.3 \mathrm{~Hz}, 0.45 \mathrm{H}$, ketal), 7.57 (dt, $J=1.7,8.8 \mathrm{~Hz}, 0.45 \mathrm{H}$, ketal), $7.50(\mathrm{dt}, J=1.7,8.8 \mathrm{~Hz}, 0.45 \mathrm{H}$, ketal), 7.40-7.18 (m, 7H, ketal), 4.70 (dd, $J=10.0,2.2 \mathrm{~Hz}, 0.1 \mathrm{H}$, enol), 4.29 (dd, $J=6.8,3.2 \mathrm{~Hz}, 0.45 \mathrm{H}$, ketal), 4.16 (dd, $J=11.5,6.8 \mathrm{~Hz}, 0.45 \mathrm{H}$, ketal), 3.87 (dd, $J=19.5,10.2$ Hz, 0.1H, enol), 3.32 (dd, $J=19.3,2.2 \mathrm{~Hz}, 0.1 \mathrm{H}$, enol), 3.22 (br d, $J=13.6 \mathrm{~Hz}, 0.45 \mathrm{H}$, ketal), 2.54-2.30 
(m, 1.4H, ketal), $2.29(\mathrm{~s}, 0.3 \mathrm{H}$, enol), $2.00(\mathrm{dd}, J=13.7,11.5 \mathrm{~Hz}, 0.4 \mathrm{H}$, ketal), 1.72 (s, 1.35H, ketal), $1.68(\mathrm{~s}, 1.35 \mathrm{H}, \mathrm{ketal}) ;{ }^{13} \mathrm{C} \mathrm{NMR}\left(100 \mathrm{MHz}, \mathrm{CDCl}_{3}\right) \delta=162.4,161.6,159.9,159.1,153.1,153.0,143.4$, $141.7,132.2,131.7,129.4,128.8,128.4,128.1,127.4,127.2,126.7,124.1,123.8,123.2,122.9,116.8$, $116.6,116.1,115.7,104.3,101.3,100.7,99.2,45.3,42.8,40.2,35.5,35.0,34.4,30.3,28.3,27.8 ;[\alpha]_{D}^{24}=$ $+19.0\left(\mathrm{c}=1.2,2\right.$-propanol) $\left(>99 \%\right.$ ee). $\left\{\right.$ Lit. $^{11}:[\alpha]_{D}^{25}=+19.1(\mathrm{c}=1.1,2$-propanol) $(>99 \%$ ee, $S$ enantiomer)\}. 
(D) References

1. Fujii, A.; Hagiwara, E.; Sodeoka, M. J. Am. Chem. Soc. 1999, 121, 5450-5458.

2. Tanaka, K.; Fu, G. C. J. Org. Chem. 2001, 66, 8177-8186.

3. Williams, J. M.; Jobson, R. B.; Yasuda, N, Marchesini, G.; Dolling, U-H.; Grabowski, E. J. J. Tetrahedron Lett. 1995, 36, 5461-5464.

4. Kanazawa, Y.; Tsuchiya, Y.; Kobayashi, K.; Shiomi, T.; Itoh, J.; Kikuchi, M.; Yamamoto, Y.; Nishiyama, H. Chem. Eur. J. 2006, 12, 63-71.

5. Duncan, A. P.; Leighton, J. L. Org. Lett. 2004, 24, 5025-5032.

6. Nishikata, T.; Yamamoto, Y.; Gridnev, I. D.; Miyaura, N. Organometallics 2005, 24, 5025-5032.

7. Halland, N.; Hansen, T.; Jørgensen, K. A. Angew. Chem., Int. Ed. 2003, 42, 4955-4957.

8. Li, H.-Y.; Robinson, A. US patent 5,856,525, 1999.

9. Robinson, A.; Li, H.-Y.; Feaster, J. Tetrahedron Lett. 1996, 37.8321-8324.

10. Demuynck, M.; Clercq, P. D.; Vandewalle, M. J. Org. Chem. 1979, 44, 4863-4866.

11. West, B. D.; Preis, S.; Schroeder, C. H.; Link, K. P. J. Am. Chem. Soc. 1961, 83, 2676-2679. 


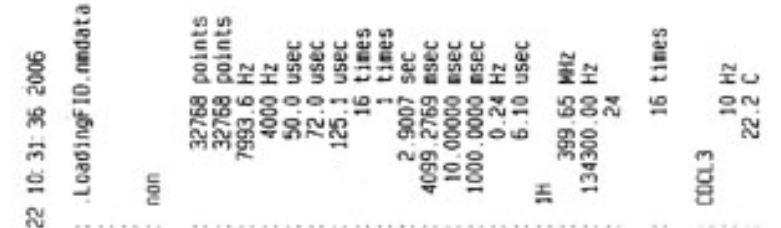

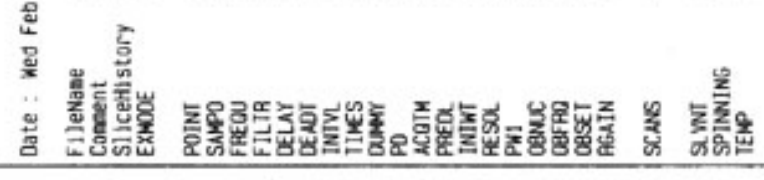

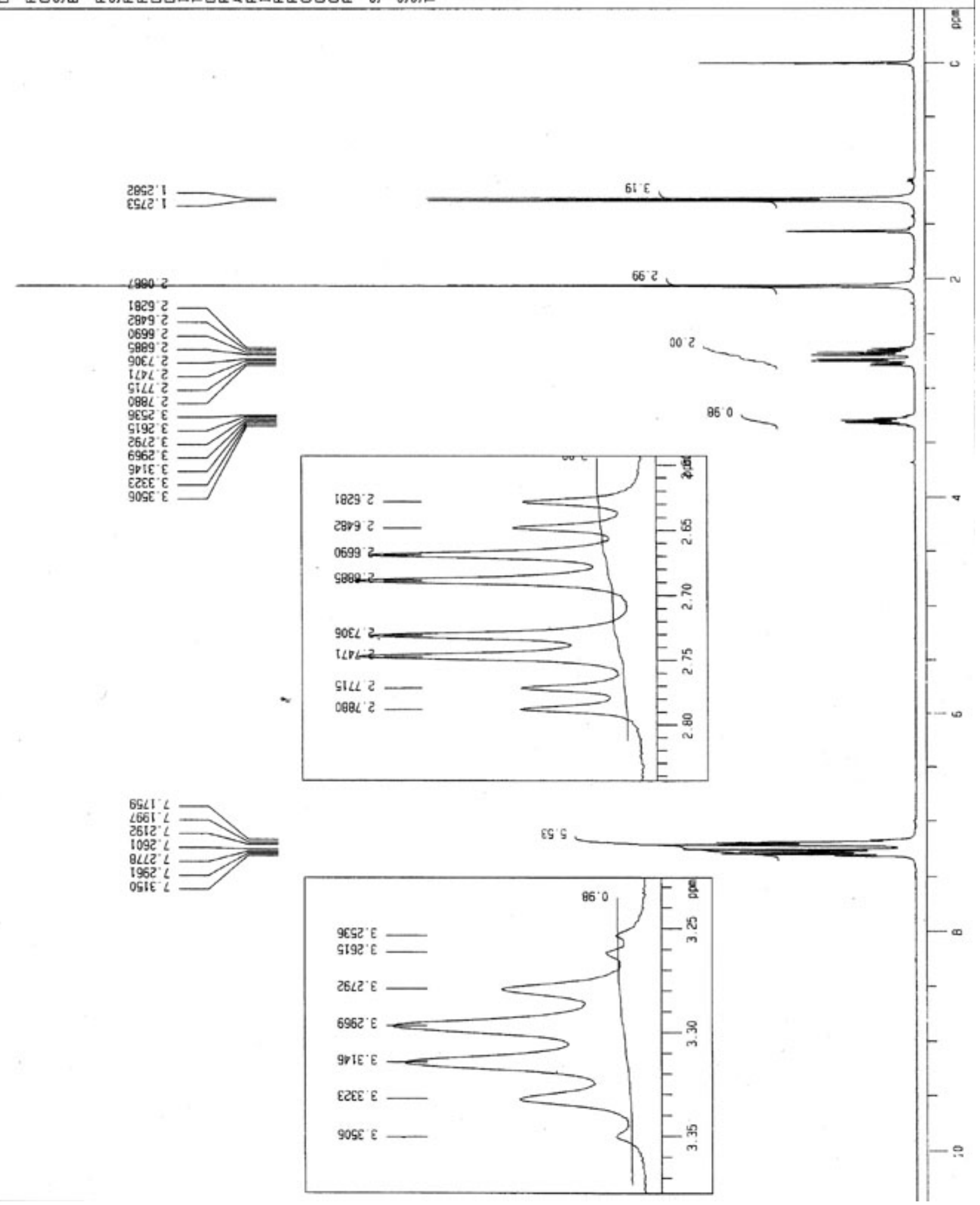



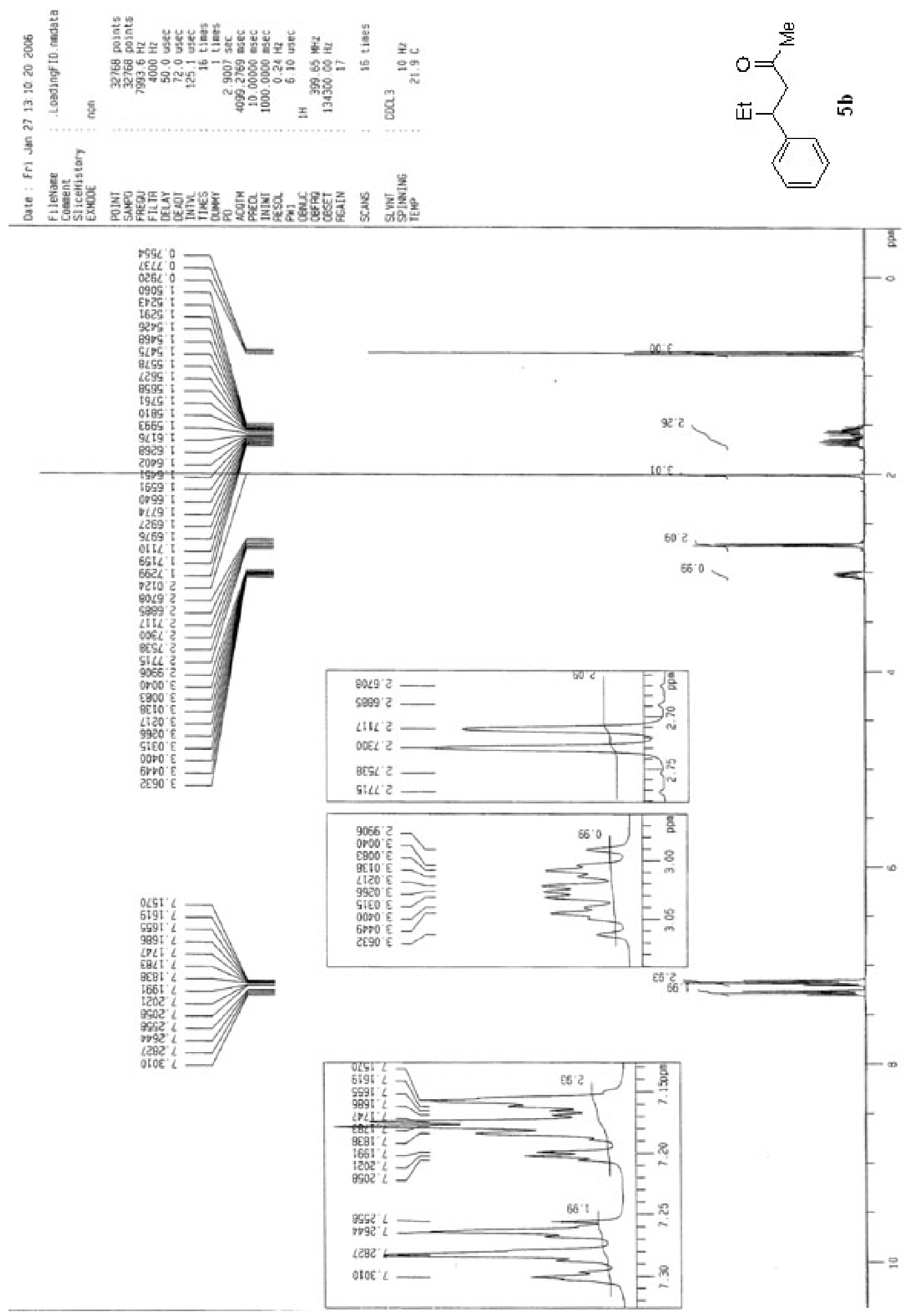

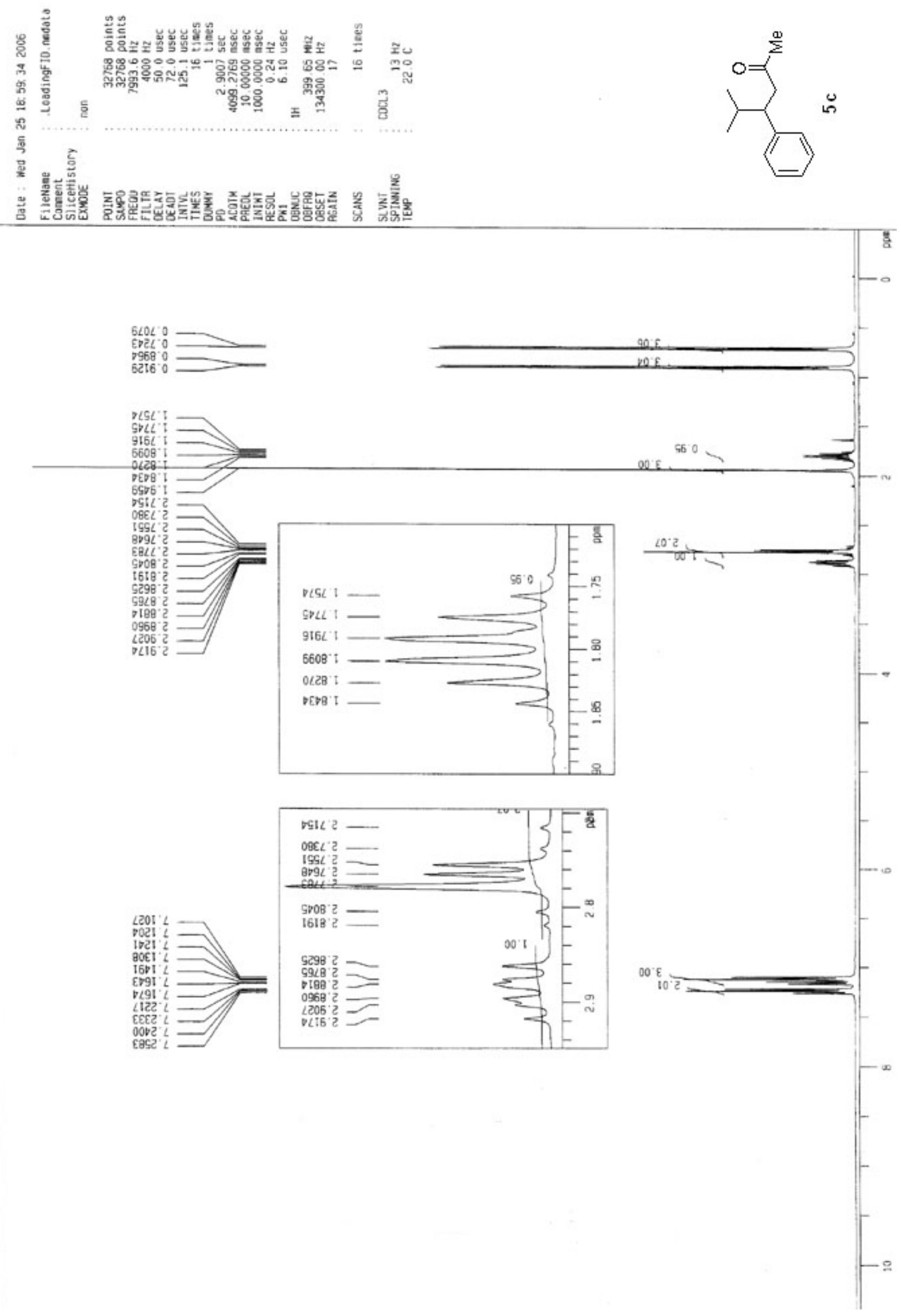

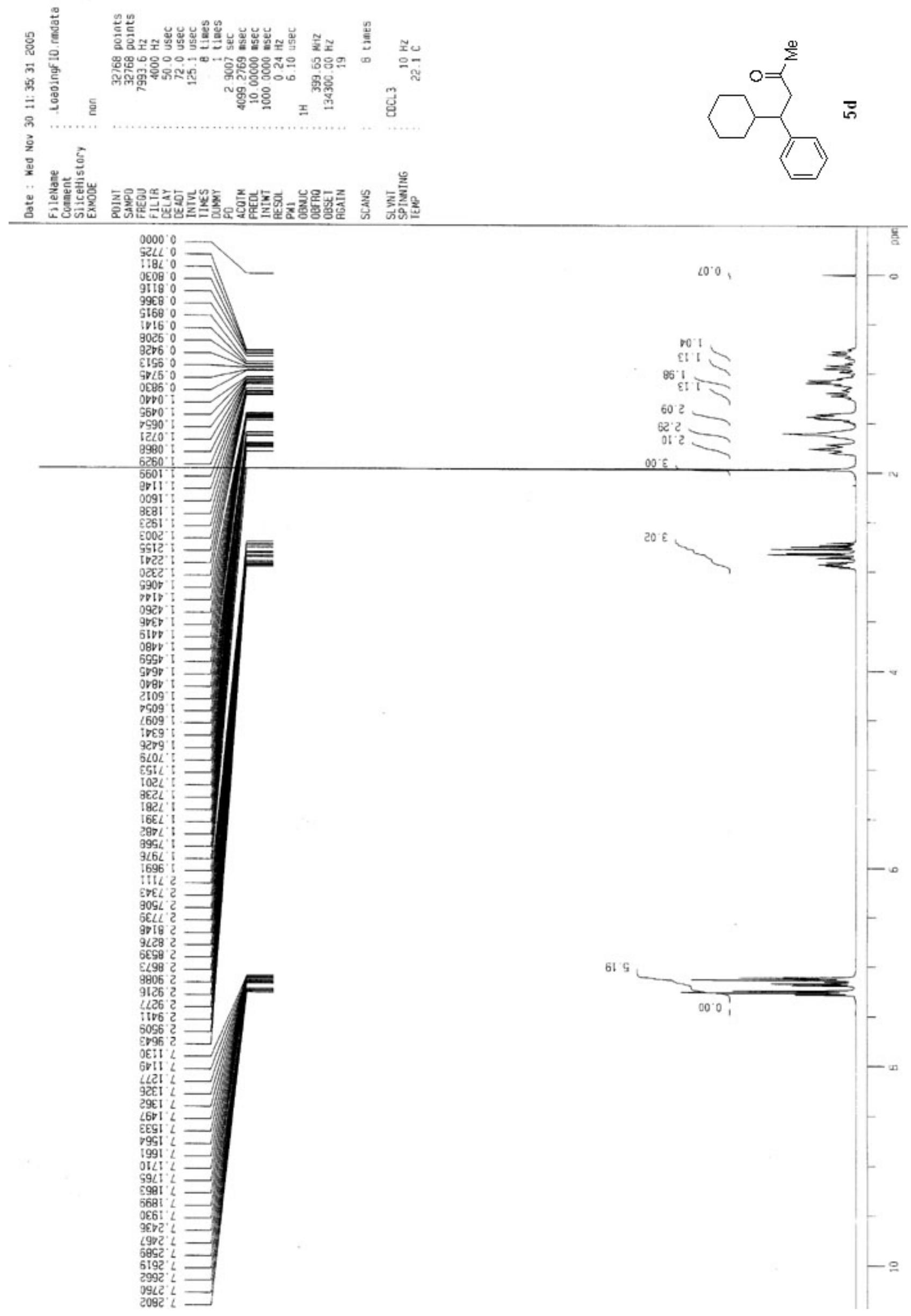


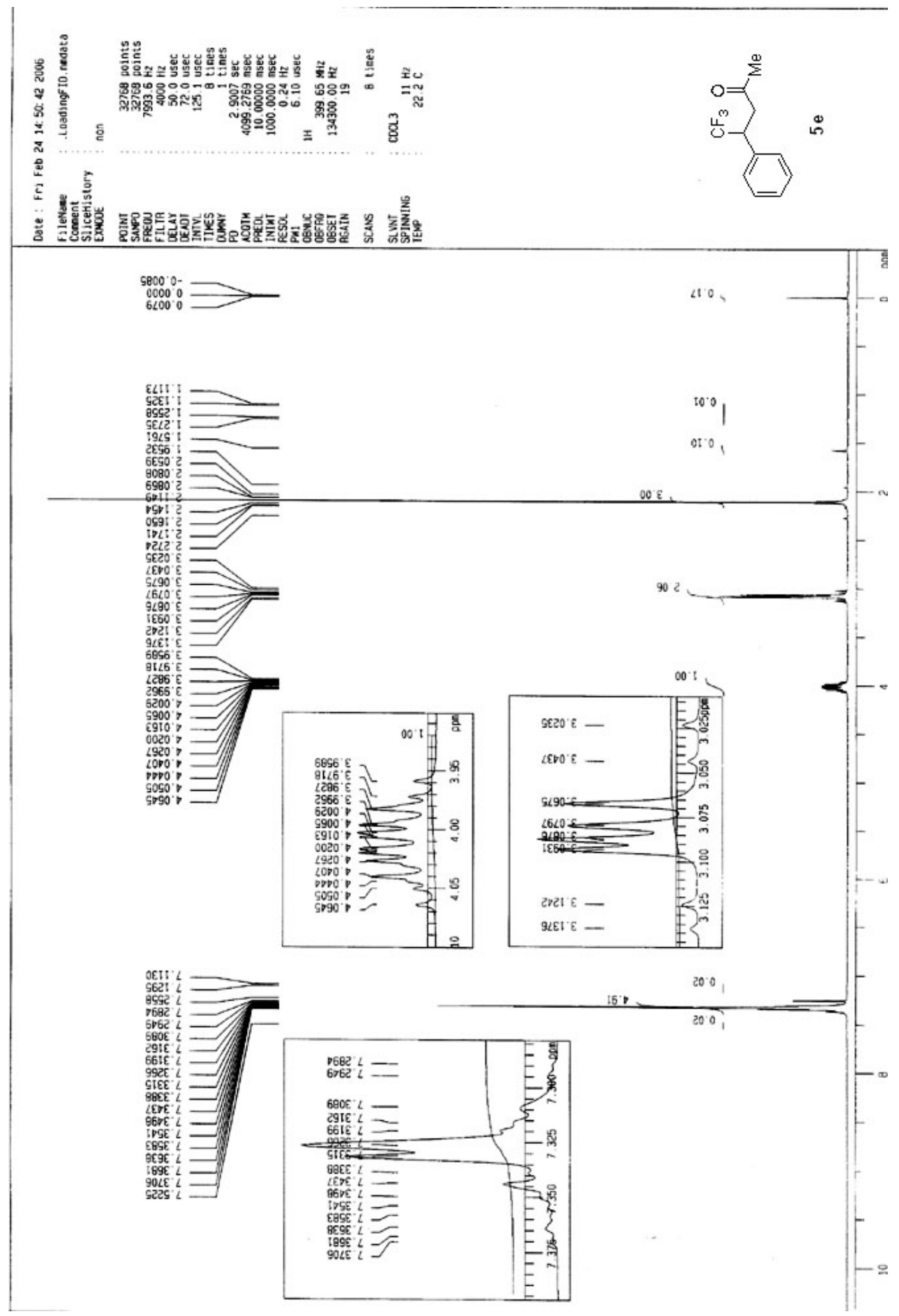




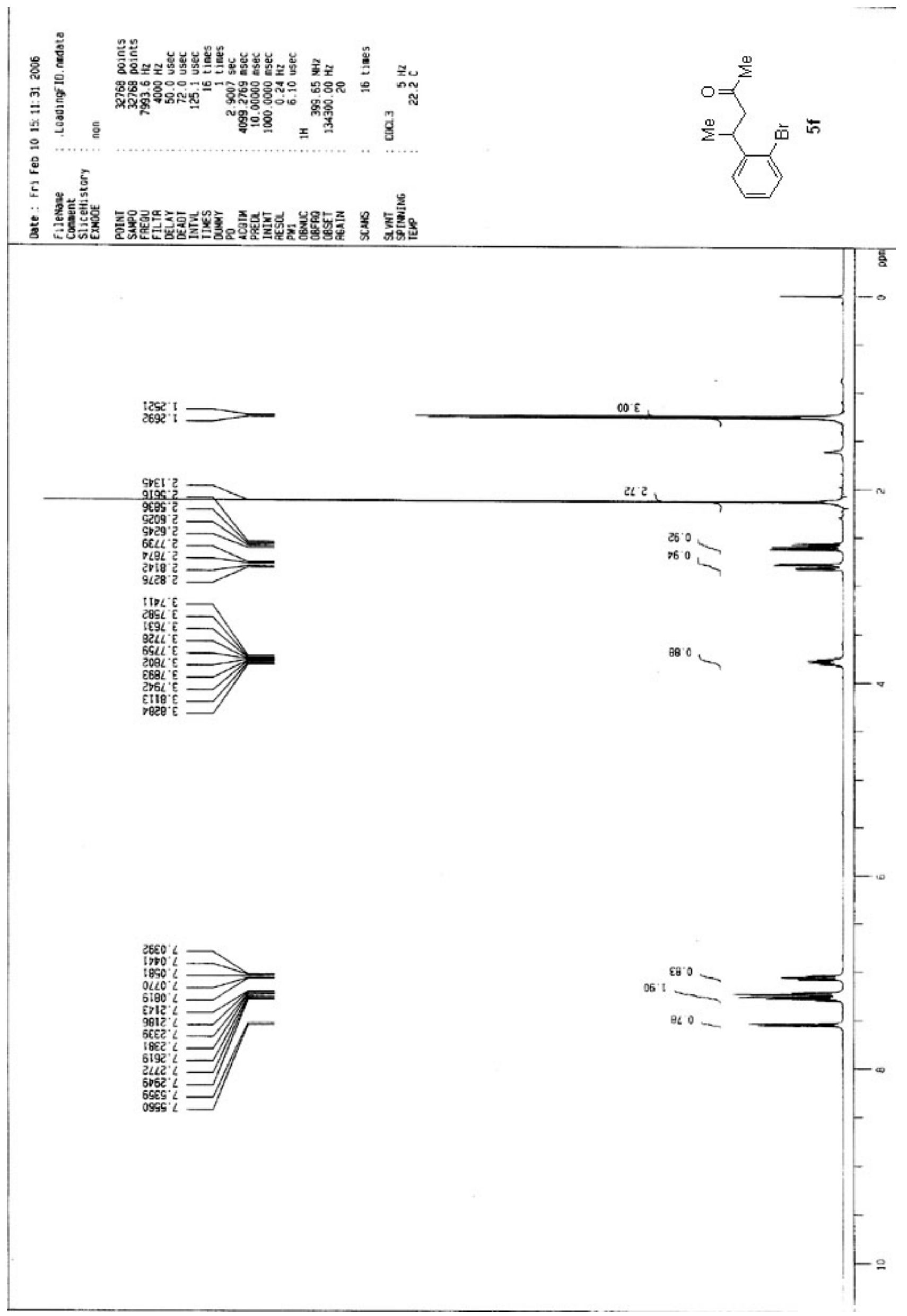




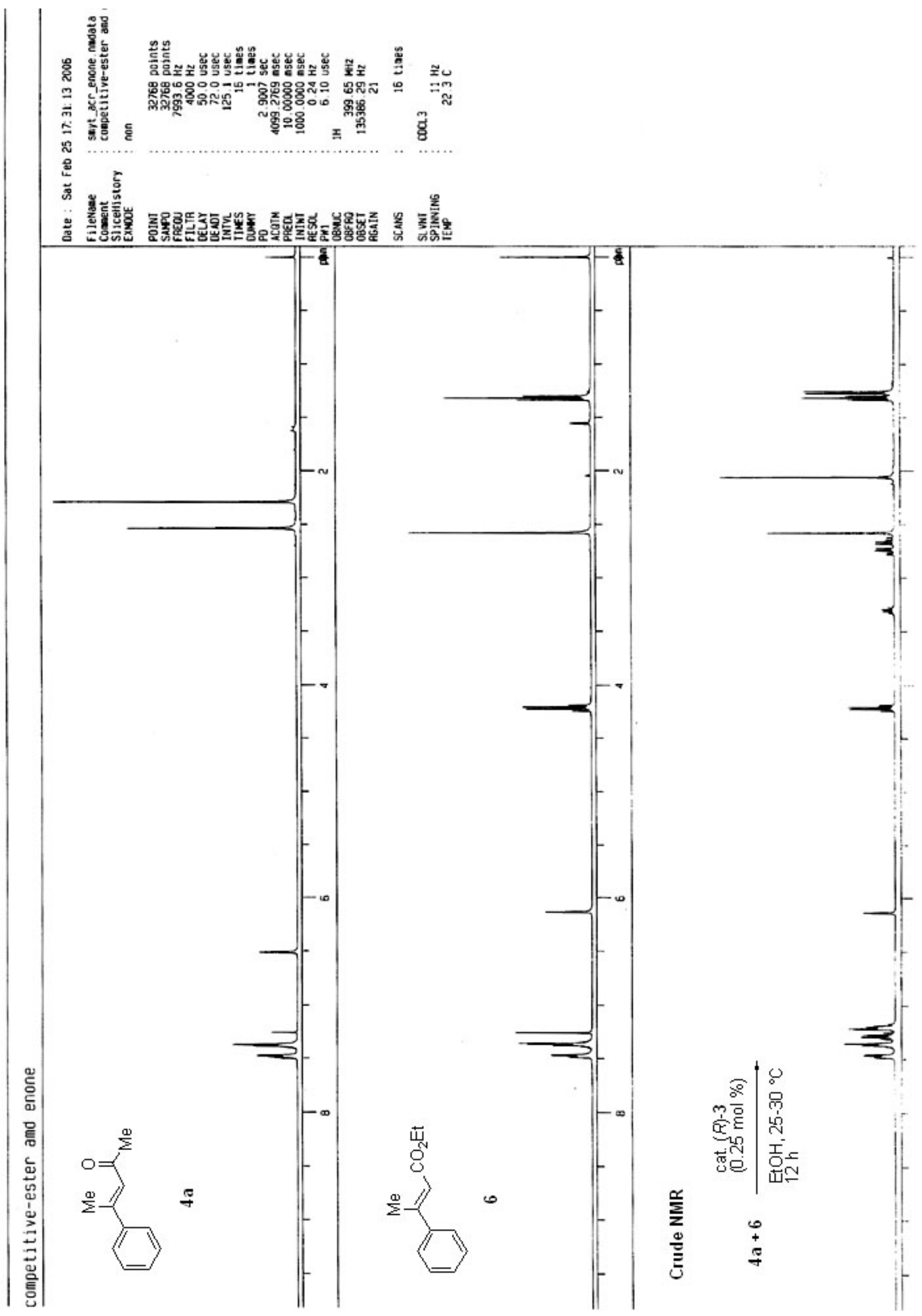




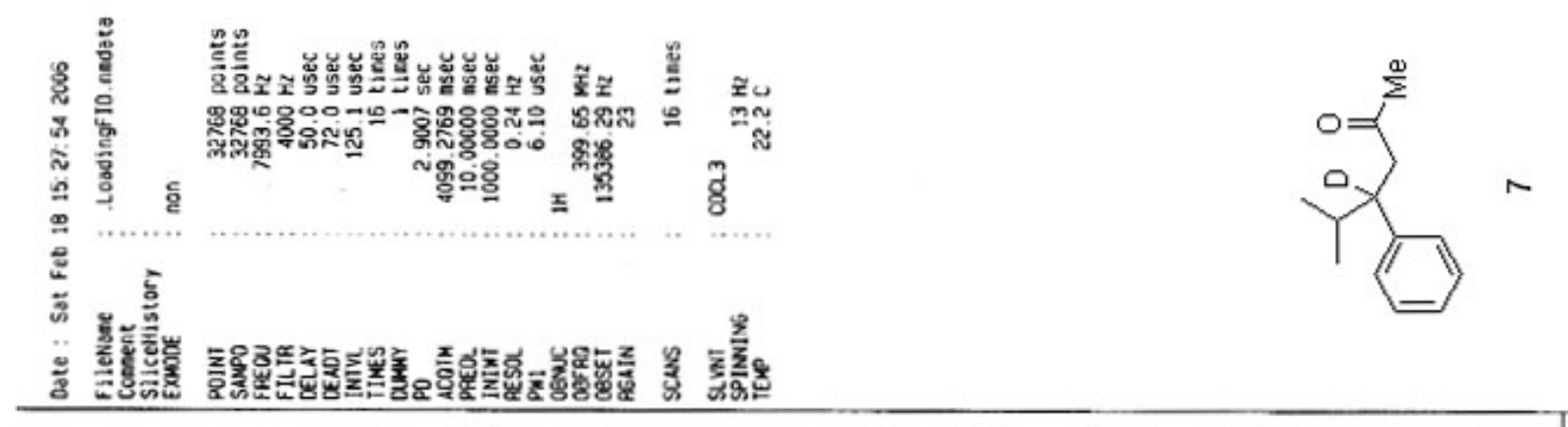

$0000^{\prime} 0$
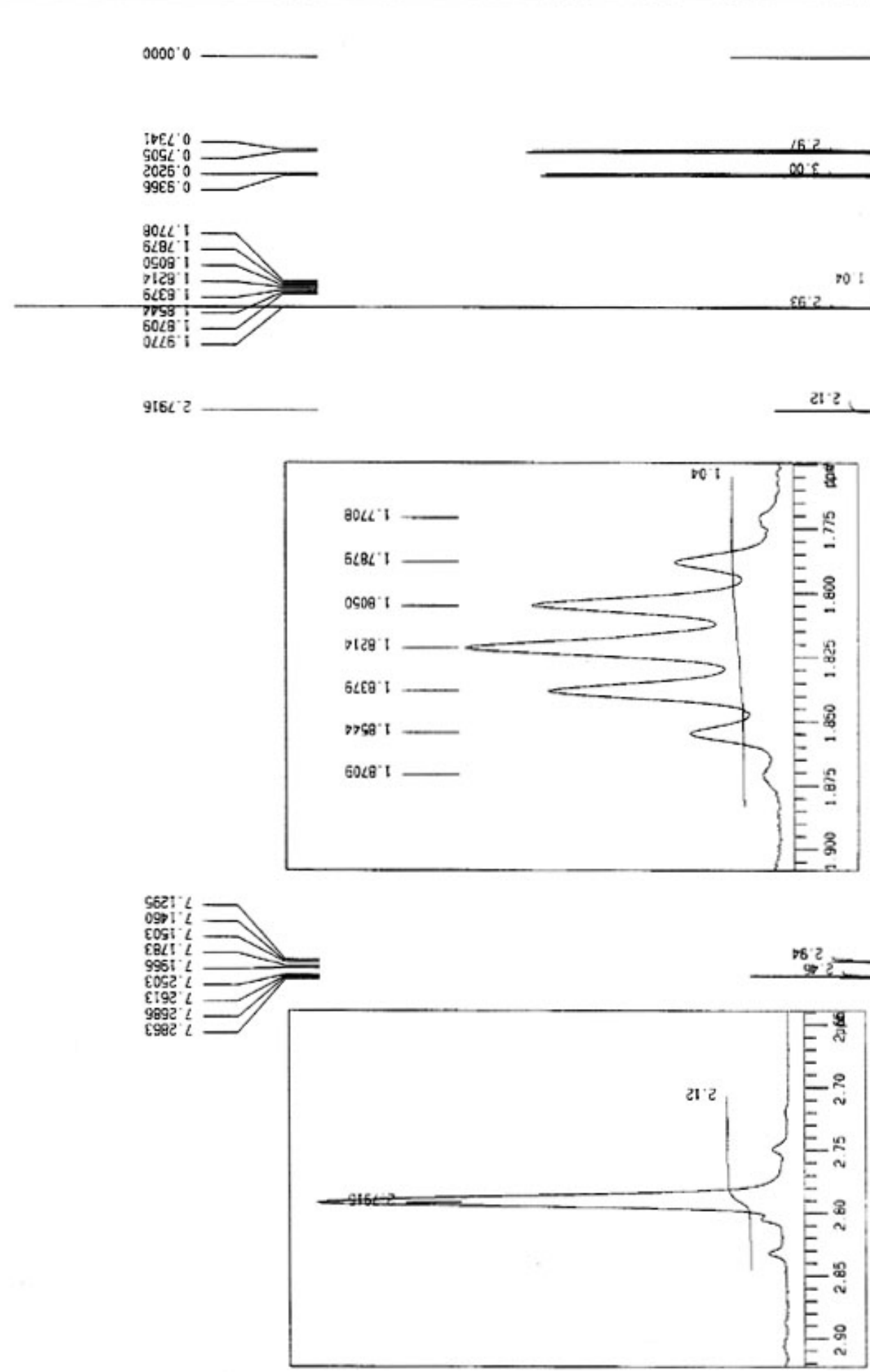

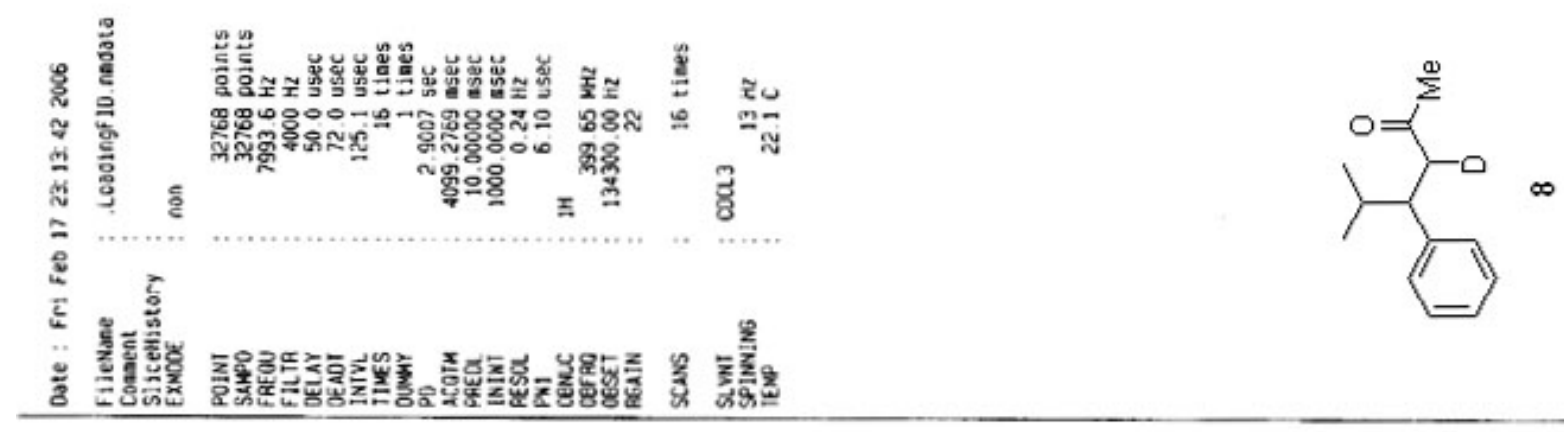

\section{a}

$0000^{\circ} 0$

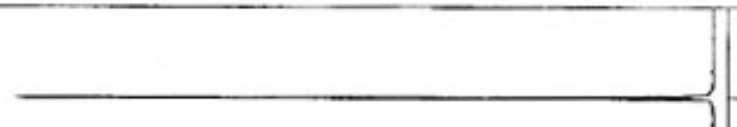

CEEL 0

S225.0

I6EБ

$1 \angle 9 L^{\circ}$

1008 .

2L:8

2768
6198

5988

6016

$25 \angle 6$

$20 D$
$L$

EDSL

ctol

$916 t$

हเEื

0908.

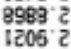

0125 '

rebs'

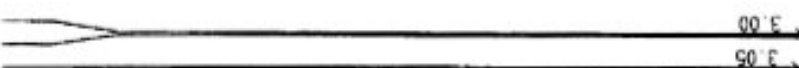




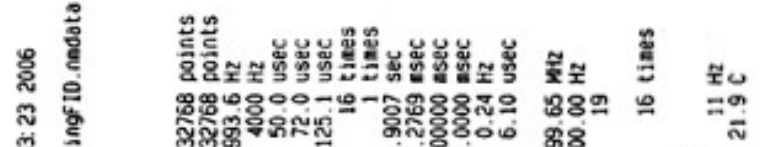

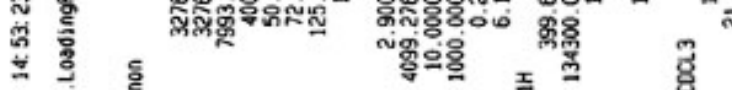

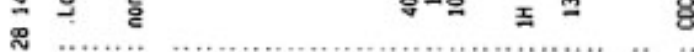

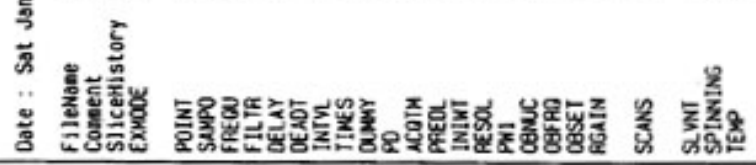
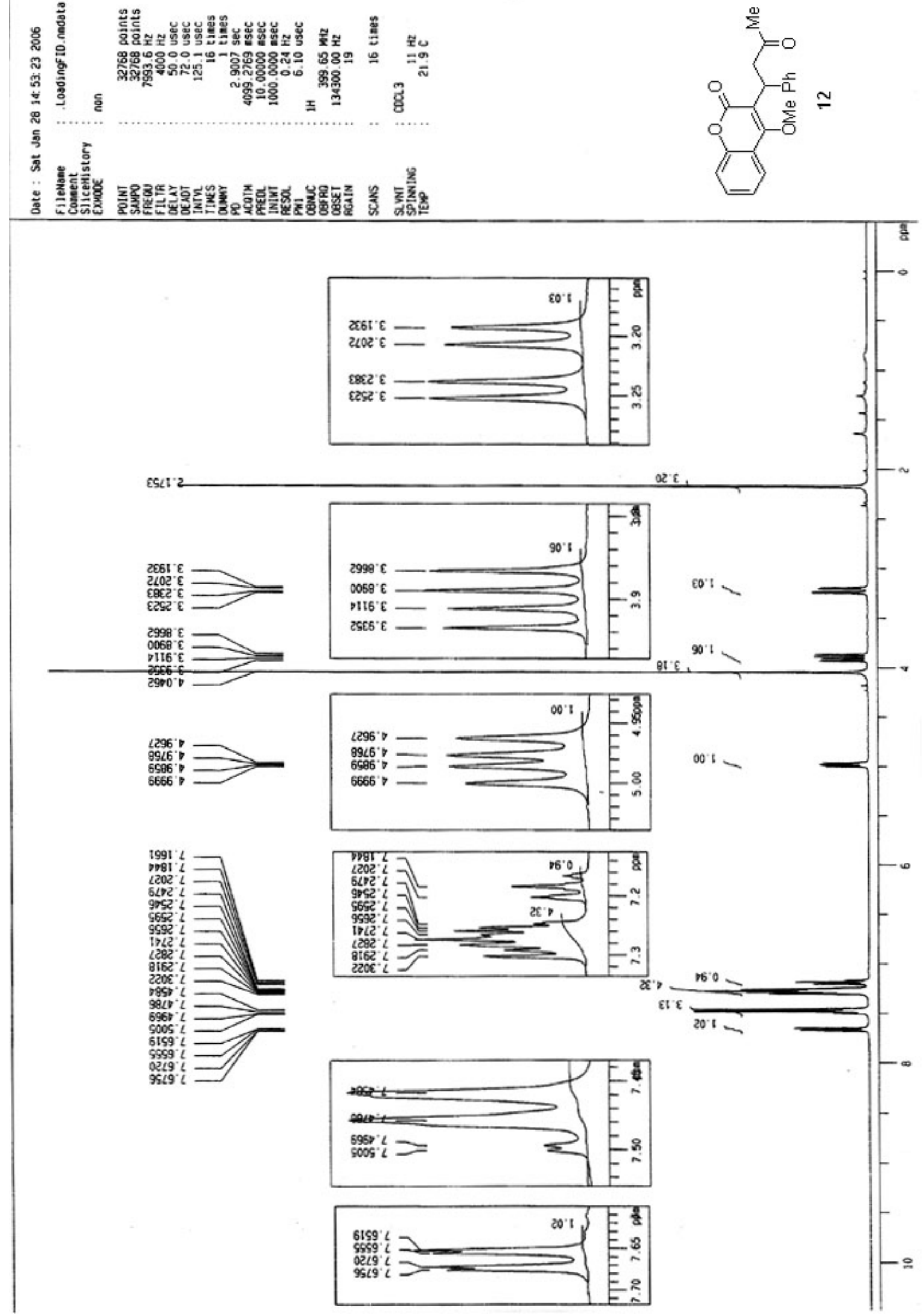

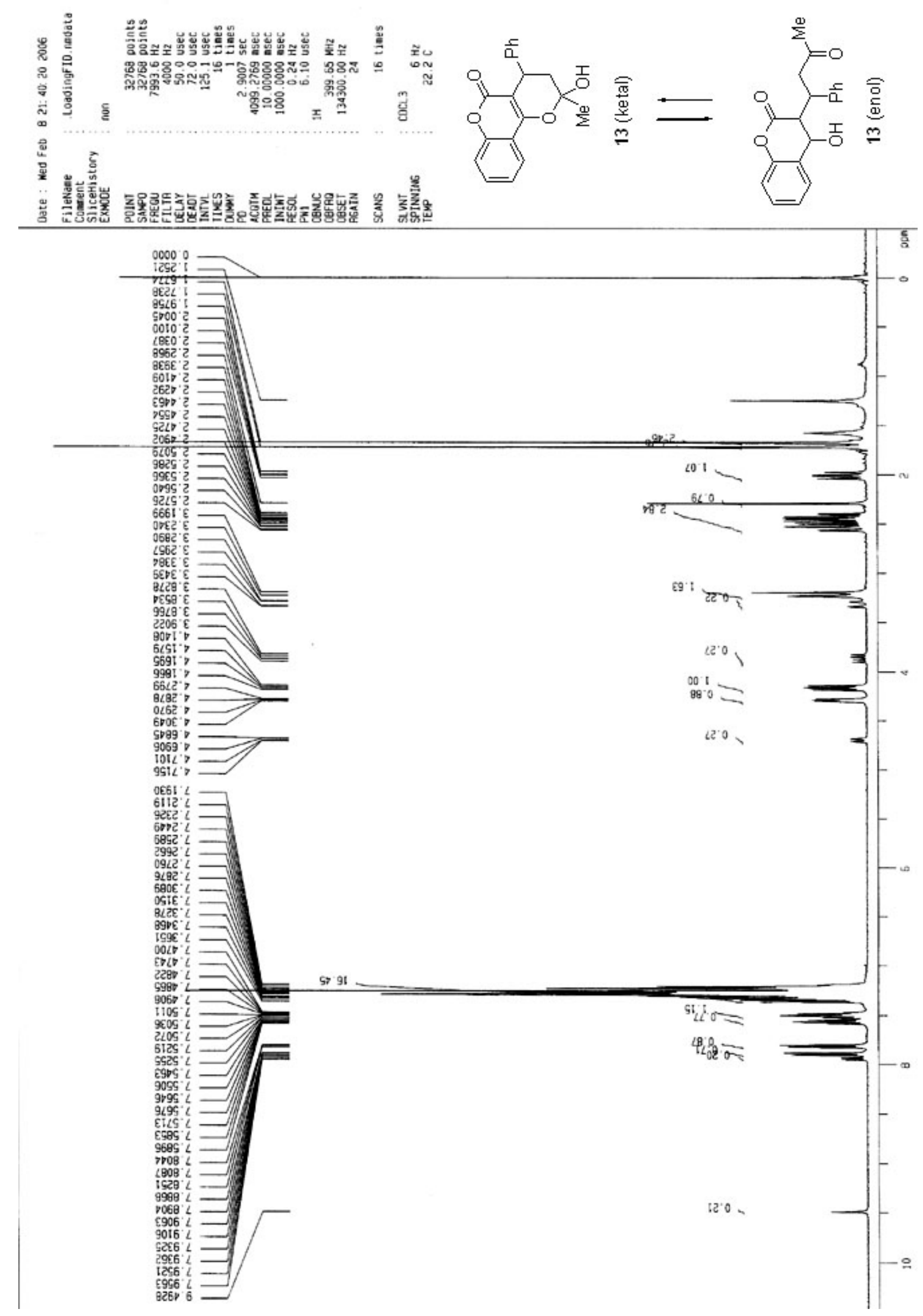
S-25 